\title{
HABILIDADES BLANDAS Y EL IMPACTO DE LA COVID-19 EN LA EDUCACIÓN SUPERIOR
}

\section{SOFT SKILLS AND THE IMPACT OF COVID-19 ON HIGHER EDUCATION}

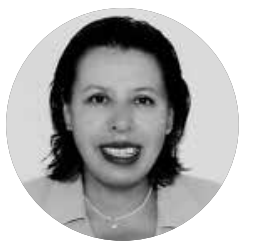

\section{Lida Vásquez-Pajuelo}

Código ORCID:

https://orcid.org/0000-0002-1241-3190

Universidad Peruana de Ciencias Aplicadas,

Lima, Perú

Correspondencia:

pcaplvas@upc.edu.pe

lidavasquez@gmail.com

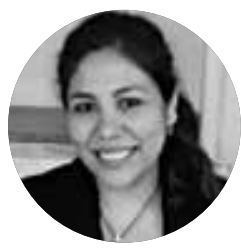

\section{Jazmín Alicia Tuesta-Vila}

Código ORCID:

https://orcid.org/0000-0001-6616-3978 Universidad Peruana del Norte, Lima, Perú Correspondencia:

jazmin.tuesta@upn.pe

jaztuesta@gmail.com

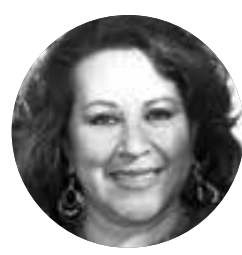

Doris Alicia Vila-Gómez

Código ORCID:

https://orcid.org/0000-0002-1855-7830

Universidad San Ignacio de Loyola, Lima, Perú

Correspondencia:

doris.vila@usil.pe

dorisvila@yahoo.comi

\section{RESUMEN}

El presente artículo de investigación busca conocer cómo fomentar el desarrollo de las habilidades blandas para afrontar con éxito los impactos en la educación superior del periodo post pandemia. El estudio se basa en la revisión exhaustiva de los trabajos actuales que se tienen a disposición sobre el conocimiento de las habilidades blandas y la promoción de su desarrollo en el estudiante. Esto permitirá perfeccionar sus habilidades blandas y ser consciente de sus propias cualidades socioemocionales. De esta manera, en el futuro podrán ser parte de una generación de profesionales capaces de tener un desenvolvimiento emocional armonioso, que incluya momentos de alta presión laboral. La educación superior debe promover entre la comunidad educativa que la conforma, en especial entre los estudiantes, las distintas alternativas de expansión de las habilidades blandas, con los consiguientes impactos positivos en su futuro mundo laboral. Entendiendo estas como habilidades que potencian la interacción de uno con el otro, fortaleciendo el trato, y mejorando las relaciones y el control emocional en el ambiente laboral, profesional y personal. La metodología aplicada en este trabajo es cualitativa y consistió en la revisión de artículos actuales y recojo de datos de distintos autores que explican lo que las "habilidades blandas" significan. Los resultados presentados en esta investigación corresponden a la inquietud de buscar un método eficaz que permita a los estudiantes desarrollarse de manera dinámica e intuitiva con la asistencia e intervención del docente, quien también debe reunir las características necesarias para ser el apoyo constante del educando. La investigación da pie a que, en el futuro, se profundice el desarrollo de las habilidades blandas en los estudiantes y sirva de base para futuras investigaciones. 
Palabras clave: habilidades blandas; impacto de la Covid-19; educación superior.

\section{ABSTRACT}

This research paper seeks to know how to promote the development of soft skills in order to face successfully the impacts on higher education of the post-pandemic period. The study is based on an exhaustive review of current literature on soft skills and the promotion of their development in students. Such promotion will allow students to improve their soft skills and become aware of their own socio-emotional qualities. In the future, accordingly, they will be able to be part of a generation of professionals capable of having a harmonious emotional development, which includes moments of high work pressure. Higher education should foster among its community members, especially among students, the different means for expanding soft skills, with the consequent positive impacts on their future employment. Soft skills should be understood as those skills that enhance interaction between one another; strengthen relationships; and improve relationships and emotional control in work, professional and personal environments. The methodology applied in this study is qualitative in nature, and consists of both, reviewing current research, and collecting data from different authors that explain what "soft skills"mean. The results presented in this study are consistent with our concern of seeking an effective method that allows faculty-assisted student development, dynamically and intuitively We stress the need for enhancing students with soft skills, whose further development should set the stage for future applied research

Keywords: Strategic planning, operative efficacy, long-term vision.

\section{INTRODUCCIÓN}

El fortalecimiento del desarrollo de las habilidades blandas en estudiantes de educación superior es un tema vigente y conlleva el planteamiento siguiente: ¿cómo se incentiva el desarrollo de las habilidades blandas en la educación superior? Sin embargo, en medio de una pandemia que impone el aislamiento social, que coloca por delante a la vida como valor más importante, dicha pregunta queda reformulada en la siguiente interrogante: ¿cómo se incentivan el desarrollo de las habilidades blandas en la educación superior en el periodo pos-pandemia?

Esta situación trae, entre otras consecuencias, el aumento del nivel de estrés, debido a que los alumnos deben estudiar y trabajar de modo sincrónico. Por tanto, es importante motivar el desarrollo de estas habilidades mediante actividades sociales, cursos online, webinars y charlas informativas, entre otros, en los que se integren dinámicas, aplicando estrategias para promover el pensamiento crítico del estudiante y fomentar la comunicación oral, como también trabajar en equipo, para que así puedan tomar sus propias decisiones y solucionar problemas. En este esfuerzo, los docentes también participan incentivando a sus estudiantes para que su aprendizaje sea más profundo y de calidad. Además, el docente ayuda a los estudiantes a consolidar, paso a paso, su autonomía, es decir, sus habilidades de autoaprendizaje y autogestión para enfrentar, mejor equipados, los desafíos del mercado laboral y el eventual contexto de la pos-pandemia que se vivirá.

Por otro lado, la realidad laboral del siglo XXI exige nuevas habilidades a los egresados de las universidades, tales como las habilidades digitales. Al respecto, recientes investigaciones demuestran que quienes poseen alta habilidad en el uso de las TIC para la gestión de información, así como para manejar dispositivos móviles, pueden desarrollar el pensamiento crítico y su capacidad de resolver problemas. Además, los estudios precisan que la realización de proyectos académicos fortalece la adquisición y desarrollo de tales habilidades en relación con el uso de las TIC. (León-Pérez, Bas, \& Escudero-Nahón, 2020).

El propósito de este artículo de investigación es indagar cómo fomentar el desarrollo de las habilidades blandas en los estudiantes para afrontar la educación superior en el periodo de la pos-pandemia.

En la actualidad, las habilidades blandas son tomadas en cuenta en distintas organizaciones humanas, como las 
empresas, en donde se valoran la inteligencia y el control emocional de los colaboradores por sus impactos en los resultados productivos. Así, además de los conocimientos técnico-prácticos, la capacitación de los trabajadores en el desarrollo de las habilidades blandas se relaciona con su capacidad para el relacionamiento social, su forma de comunicar, sus aptitudes y personalidad, permitiendo un nivel alto de competitividad personal, grupal y empresarial.

En este artículo, se resalta que la salud mental en los estudiantes es de suma importancia para enfrentar la pos-pandemia y que se mantiene a través del desarrollo de las habilidades blandas. A su vez, estas habilidades están relacionadas con el desempeño académico al permitirles ser competentes y/o versátiles en diversas situaciones. Asimismo, es necesario comprender que los profesores tienen un rol de liderazgo en este proyecto y necesitan desarrollar las competencias necesarias para influir en el estudiante de una manera positiva (Flores-Guerra, 2018).

\subsection{Las habilidades blandas}

En 1959, la Marina de Guerra de los Estados Unidos de América (US Navy) implementó un programa de capacitación para sus integrantes. Para tal fin, usó un método para medir, mediante pasos o procesos, las competencias y habilidades necesarias para una mejor integración y mejor desempeño de sus miembros en su especialidad militar. Estas competencias desarrolladas no tenían una palabra que conceptúe la idea, tan solo era llamadas "habilidades necesarias para mejorar el desempeño laboral, [que] carece[n] del contacto con máquinas...."

El origen del uso formal de la expresión "habilidades blandas" se ha identificado en un manual de entrenamiento del ejército estadounidense de 1972, se da a conocer las "habilidades blandas" (del inglés Soft Skills). El psicólogo Nicholas Humphrey afirmó célebremente que es la inteligencia social la que define a los humanos más que la inteligencia cuantitativa. Muchas empresas dan hoy preferencia a las habilidades blandas de sus empleados. (referido en Newman, Chutes \& Ladders, 2006).

Posteriormente, en la década de los 90, aproximadamente en 1996, la expresión "habilidades blandas" en la educación, comienza a distinguirse en contextos de gestión y emprendimiento; adquiere diferentes nombres, tales como habilidades del siglo XXI, habilidades no cognitivas, habilidades socioemocionales o blandas (Murnane y
Levy, 1996). Cabe resaltar que registra términos como competencias para la empleabilidad o habilidades laborales, habilidades relacionales o transversales. Del mismo modo, Goleman (1998), cataloga a estas habilidades como inteligencia emocional y considera que esta determina la capacidad de adaptabilidad frente a situaciones nuevas suscitadas en los diferentes contextos de la vida del hombre: laboral, educativo, personal, etc.

Las habilidades blandas o soft skill son definidas como aquel grupo de destrezas adquiridas por la persona, en este caso del estudiante y docente, y que facilitan la optimización de su propio desempeño, tanto en el ámbito académico-profesional, laboral, emocional, psicológico como en el ámbito personal (Duckworth y Yeager, 2015; Siqueira, 2017). Cabe destacar que estas habilidades se complementan con otras, calificadas como habilidades duras (Marrero, 2018). Respecto a los estudiantes las habilidades blandas, consideradas también como habilidades socioemocionales, habilidades no cognitivas, inteligencia emocional, competencias para la empleabilidad, habilidades laborales, habilidades relacionales o transversales; son cruciales para el desarrollo académicoprofesional y personal del docente universitario, pues es preciso que estos formen a estudiantes para la vida a partir de capacidades como saber escuchar y comunicar, por ende, retroalimentar de forma positiva; pensar de forma crítica; liderar a partir de la empatía. Y respecto del desempeño docente en el nivel superior de educación y su evaluación, se puede concluir que se realiza desde variados criterios, tal es el caso de competencias útiles para el desarrollo del proceso de enseñanza: saber planificar, poseer comunicación efectiva, manejar las herramientas tecnologías, aplicar una metodología coherente al contexto, interactuar de forma asertiva con los estudiantes, tutorizar de forma pertinente, fomentar el trabajo colaborativo e individual y autorregular (referido en Rodriguez, Rodríguez, \& Fuerte, 2021).

\subsection{El impacto de la Covid-19}

Desde su llegada, el coronavirus y la pandemia generada han trastocado la vida de todos en una escala mundial. Como se conoce, los coronavirus generan desde afecciones respiratorias leves, como el resfrío común, hasta formas más severas, como el Síndrome Respiratorio de Oriente Medio (MERS, por sus siglas en inglés), y el Síndrome Respiratorio Agudo Severo (SARS, por sus siglas en inglés). El actual brote de coronavirus está generando 
una afección conocida como COVID-19 (García, 2020), y ya ha afectado a casi cincuenta millones de personas en todo el mundo. Sin embargo, no se conoce todavía las secuelas de largo plazo. Al respecto, en una entrevista en la Actualidad TV, el director general de la Organización Mundial de la Salud (OMS), Tedros Adhanom Ghebreyesus, ha manifestado que dichas secuelas presentarían "una preocupante variedad de síntomas que pueden cambiar con el tiempo y pueden afectar a cualquier sistema corporal" (referido por Izquierdo, 2020, p. 1).

Hasta el momento no hay una cura a esta enfermedad, siendo la única alternativa la inmunización. Cuatro de las 10 vacunas más avanzadas (Fase III) se desarrollan en China, mientras que las otras son de las farmacéuticas estadounidenses, Janssen (Johnson \& Johnson), Novavax, Pfizer y Moderna; a las que se suma la británica AstraZeneca, en colaboración con la Universidad de Oxford. En el Perú ya se están probando las vacunas de Sinophram en asociación con la Universidad Mayor de San Marcos y la Universidad Peruana Cayetano Heredia.

\section{MÉTODOS Y MATERIALES}

En la presente investigación se ha empleado la metodología cualitativa, enfocada en el análisis documental y la revisión sistemática de fuentes de información actuales, como artículos científicos, entre otros, usando como técnica, la lectura comprensiva, de textos descriptivos y explicativos con contenido especializados y científicos.

\subsection{Las habilidades blandas versus el impacto de la COVID-19}

Como se precisó, este estudio está basado en la revisión de artículos de publicaciones recientes y recojo de datos de distintos autores que explican el concepto de las "habilidades blandas y el impacto de la COVID-19 en la educación superior" y se desarrolla desde las perspectivas de la psicología y la educación. Cabe precisar que la investigación se realiza durante este año de pandemia. El presente estudio, se basa en el esquema de elaboración de las autoras presentado en la Figura 1 y que recoge los planteamientos de Aguerrevere et al. (2020).

\section{Figura 1. Habilidades blandas vs. impacto de la COVID-19}

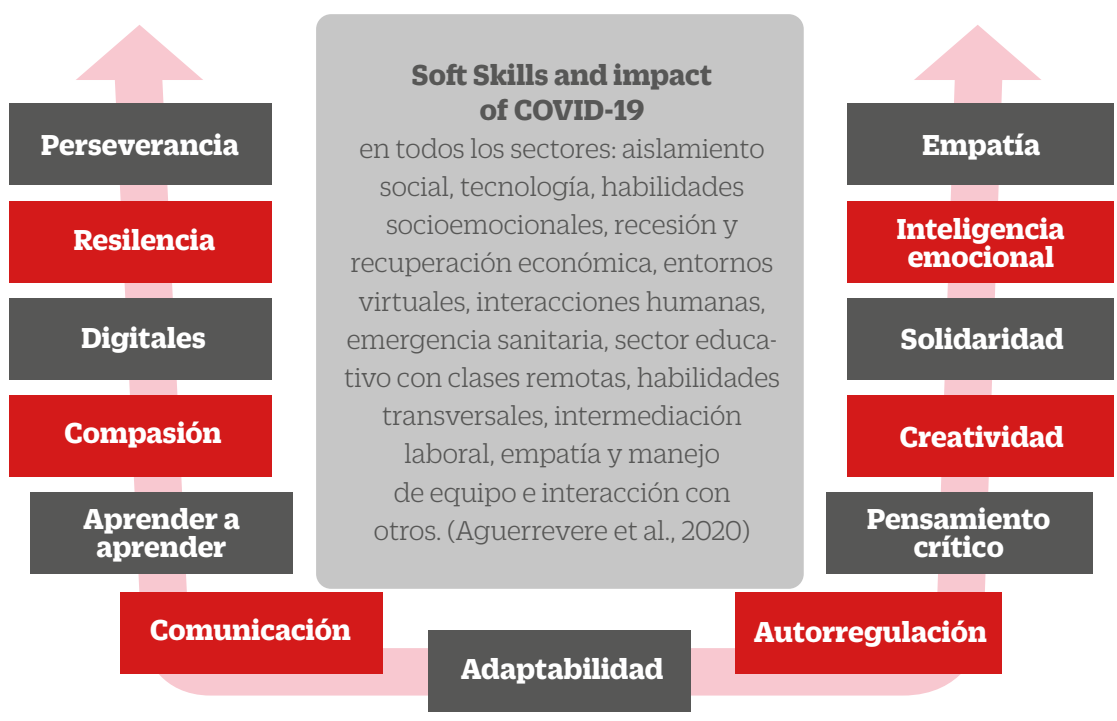

Fuente: Adaptado de Aguerrevere et al., 2020. 


\subsection{Revisión de habilidades blandas}

En primer lugar y tal como lo consigna un artículo en la versión chilena del portal Universia ("Habilidades blandas: qué son", 2020), las habilidades blandas se caracterizan por permitir que la persona que las posee tenga un relacionamiento más efectivo en los distintos escenarios donde le toque desenvolverse. Por lo tanto, tienen que ver con la puesta en práctica integrada de aptitudes, rasgos de personalidad, conocimientos y valores adquiridos; destacándose como características de ellas a las siguientes:

- Adaptabilidad: adaptarse a los cambios y mantener la organización actualizada, con flexibilidad y salida de la zona de confort para superar miedos, cambios u obstáculos; las empresas, tal y como lo hace la sociedad, están en cambio constante.

- Autorregulación: conocerse a sí mismo, sus fortalezas y debilidades y confiar en su propio talento.

- Pensamiento crítico: aprender a pensar y elaborar su propio juicio.

- Habilidades comunicativas: ser claro a la hora de comunicar cualquier eventualidad, ya sea buena o mala, es fundamental; tener una buena comunicación con su equipo de trabajo es fundamental; saber comunicar sus ideas y recibir las de los colaboradores es parte clave. - Creatividad: tener inventiva propia, pensar fuera del rebaño y generar ideas innovadoras es una de las aptitudes más buscadas; capacidad para crear, innovar e imaginar.

- Responsabilidad: se trata de saber asumir responsabilidades, cumplir con plazos, llegar en hora, respetar los tiempos de los demás, etc.

- Honestidad: es fundamental ser honesto tanto a la hora de reclamar reconocimiento y emitir quejas, como de reconocer errores y asumir responsabilidades sin apuntar a otros.

- Proactividad: si surge un problema, lo que hay que hacer es tomar las riendas del asunto y encontrar soluciones propias sin estar constantemente molestando al jefe.

- Resiliencia: es un término acuñado por la psicología moderna y que se identifica con la capacidad de tornar situaciones complicadas y traumáticas en importantes enseñanzas de vida.

- Trabajo en equipo: se trata de tener la tolerancia y diplomacia suficientes como para lidiar con distintos tipos de personalidades; "la unión conlleva el éxito”, por lo que saber trabajar en equipo es hoy en día una habilidad indispensable para todos.

- Empatía: ponerse en el lugar del otro es primordial, ya no solo para cargos con cierta responsabilidad, sino también para el resto de los miembros del equipo;.existen dos tipos de empatía: cognitiva y afectiva, y las dos nos ayudan a ponernos en el lugar de la otra persona. No obstante, existe una gran diferencia entre ellos. El primero, puede fomentar conductas manipuladoras y servir como herramienta para herir a los demás, mientras que, el segundo tipo de empatía, por su parte, estimula la sensibilidad emocional y podría ser el camino para trabajar medidas anti-bullying.

\subsection{Las habilidades blandas en el trabajo}

De acuerdo con Universia ("Habilidades blandas: qué son", 2020), dentro de las habilidades más destacadas, tanto en el ámbito laboral, como en el universitario, tenemos a:

- Trabajar en equipo y fomentar un sentido de pertenencia: esta propuesta asume que los seres humanos necesitamos sentirnos parte de algo y asumir una identidad común, como fundamento a la hora de tomar decisiones en conjunto

- Establecer metas comunes: siguiendo en línea con el punto anterior, los objetivos a alcanzar por el equipo deben ser definidos con claridad desde un primer momento. - Construir confianza: lo normal en los grupos de trabajo es que convivan personalidades dispares, por lo que la clave para gestionarlas es construyendo confianza entre sus miembros: cada uno debe aprender a ser tolerante y conocer las virtudes y defectos de los demás.

- Promover la negociación: el camino más fácil cuando se generan dificultades de convivencia es echar culpas e intentar sacar ventaja; pero nunca se llegará a un resultado bueno si no se fomenta la empatía y se promueven las negociaciones del tipo "ganar-ganar" o "aprender a aprender".

\subsection{Las habilidades blandas en el emprendimiento}

En el emprendimiento, las habilidades más importantes serían: fijarse un objetivo, perseverar en el trabajo y aprender a fracasar. Según la un artículo en la revista Forbes ("Así ha afectado", 2020), estas habilidades la tendrían todos los emprendedores más famosos, entre los que destacan: Bill Gates, fundador de Microsoft y responsable de todos los avances informáticos y tecnológicos que hemos experimentado en las últimas décadas; Paul Allen, mano derecha de Gates en Microsoft y actor activo en Vulcan Ventures, Dreamworks, Priceline GoNet y Oxygen; Steve Jobs, fundador de Apple y reconocido por su carácter creativo e innovador; Stephen Wozniak, compañero de Jobs en Apple y verdadero cerebro detrás de la tecnología que revolucionó al mundo; Jeff Bezos, fundador de Amazon, la 
empresa de comercio electrónico más grande del mundo y quinto hombre más rico del mundo; Larry Page, creador de Google, buscador web más usado del mundo; Serguéi Brin, compañero inseparable de Page en la creación de Google;,Mark Zuckerberg, creador de Facebook, la red social en internet más importante del mundo; Walt Disney, (dibujante, creador de Mickey Mouse y cara de una de las marcas más poderosas de todos los tiempos; Henry Ford, fundador de Ford Motor Company y revolucionario de la industria automotriz.

\subsection{Impacto en la calidad educativa universitaria peruana en tiempo de cuarentena}

En el Perú, una porción importante de las entidades educativas a nivel superior ha tenido que o está en proceso de trasferir a sus estudiantes presenciales hacia una modalidad virtual para no paralizar su formación académica. Por su parte, el Ministerio de Educación diseñó una modalidad de educación virtual orientada a los escolares de primaria y secundaria, al considerar que la protección de la salud de sus educandos frente a la COVID-19 era un reto para la sociedad en general, así como el desarrollo de sus habilidades blandas, en especial (Barrios, 2020, pág. 1). Inga Arias (2020), menciona que la universidad peruana es todavía muy conservadora: recién se están trabajando currículos y sílabos por competencias. Lamentablemente, este proceso se hace sin mucha preparación, pues la transferencia de información se sigue asumiendo como "conocimiento" y de allí que se siga hablando de "dictado de clases" (p. 1). Esto choca con proyectos modernizadores y actualizadores, al que se suman los presupuestos modestos para financiar innovaciones.

En la misma línea, el Instituto Internacional para la Educación Superior en América Latina y el Caribe (IESALC) y la UNESCO han destacado la preocupación de los distintos Gobiernos para implementar programas de educación virtual, manteniendo la calidad de los mismos.

En un artículo publicado en el diario El Peruano ("Sunedu e Indecopi piden", 2020), se indica que "en el Perú, el 38\% de universidades nacionales no pudieron implementar educación virtual desde inicio del confinamiento hasta junio del 2020" (citado en Piñero, Esteban, Rojas, \& Callupe, 2021, p.125)

Las autoras, se basan en el esquema de elaboración de su autoría en la Figura 2 y que recoge los planteamientos de la UNESCO (Sistema de Información de Tendencias Educativas en América Latina, 2020).

\section{Figura 2. Educación a distancia, virtual y online}

Proceso formativo que se afianza en materiales físicos que la institución educativa entrega para realizar el curso, como separatas, libros, o material multimedia a través de $\mathrm{CD}$ u otros. Entregados directamente al estudiante. En este tipo de formación la comunicación con el profesor o tutor suele ser por correo electrónico, carta o incluso por la vía telefónica.

(Figueroa Diaz, 2020).

\section{Educación a} distancia
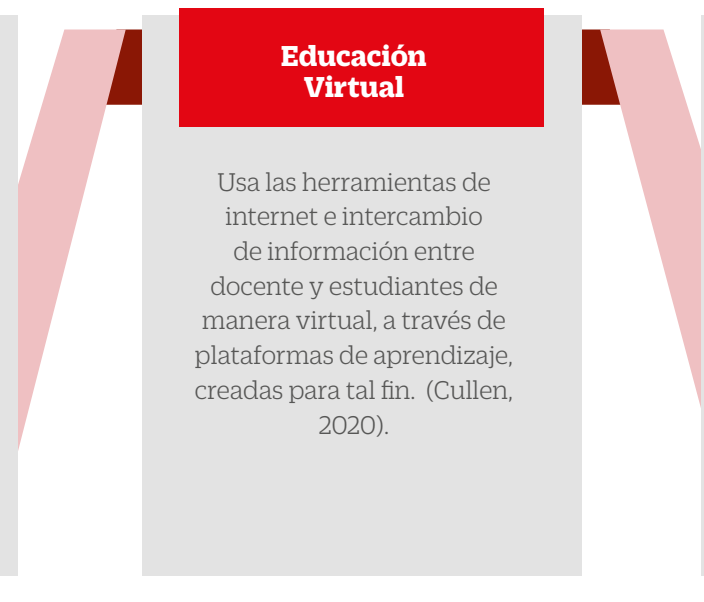

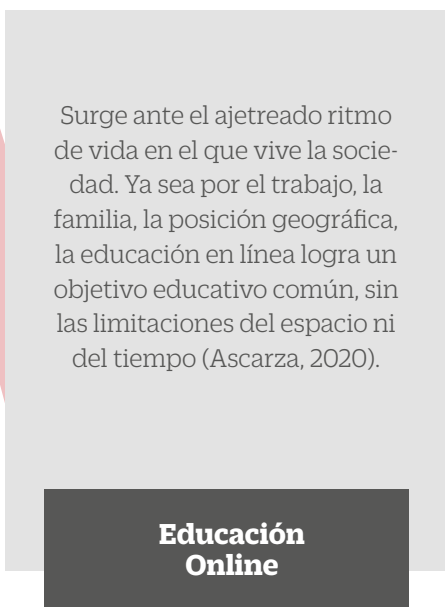

Fuente: Adaptado de Sistema de Información de Tendencias Educativas en América Latina (2020). 


\subsection{La percepción de la educación}

Benjumea destaca que la educación tecnológica viene siendo incluida como parte indispensable de las reformas educativas en varios países. Asimismo, reconoce el esfuerzo de los países por aplicarla desde el nivel preescolar hasta el superior, teniendo como fundamento al modelo de pensamiento que relaciona el "ser" con el "hacer". De esta manera, los estudiantes alcanzarán una formación integral que facilite las herramientas para la comprensión del mundo digital y logren habilidades, destrezas y aptitudes perfeccionando su razonamiento, la creatividad, organización y planificación. (citado en Hurtado Talavera, 2020a, pág.141)

En la Figura 3, las autoras proponen un esquema de su autoría, el mismo que recoge los planteamientos de Hurtado Talavera, (2020b).

\section{Figura 3. Cambio de metodología de presencial a virtual}
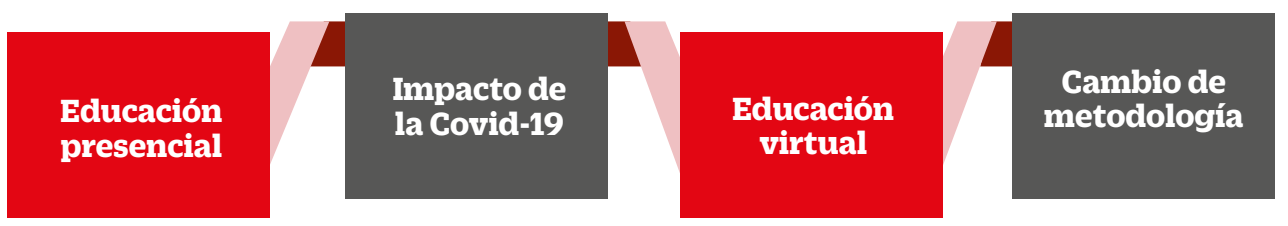

Fuente: Adaptado de Hurtado Talavera (2020b).

\subsection{Migración de las habilidades blandas a las habilidades transversales}

Las personas del siglo XXI necesitan estar equipadas con las habilidades transversales que les permitirán aprender y adaptarse a circunstancias cambiantes a lo largo de toda su vida, integrar la multiculturalidad que acarrean los procesos migratorios e identificar oportunidades de crecimiento en las diferencias. Asimismo, se requiere de personas comprometidas con dejar un mundo mejor y más próspero para las próximas generaciones y que tomen conciencia de que para progresar es importante potenciar la contribución individual y colectiva (trabajo en equipo).

En la Figura 4, las autoras proponen un esquema de su autoría que recoge los planteamientos de Mateo-Berganza et al. (2019).

\section{Figura 4. Migración a las habilidades del siglo XXI}

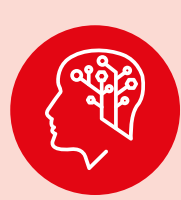

Inteligencia artificial: Comunicación; Creatividad; Digitales; Ética; Manejo del tiempo; Pensamiento crítico; Resolución de problemas; Trabajo en equipo

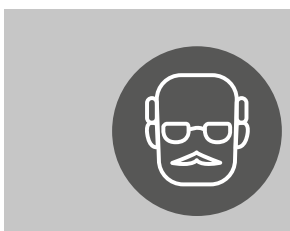

Envejecimiento: Adaptabilidad; Aprendizaje continuo; Auto-regulación; Emprendimiento; Flexibilidad; Perseverancia; Resiliencia

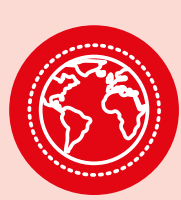

Cambio climático:

Compromiso; Eficacia; Respeto; Responsabilidad

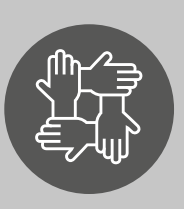

Diversidad: Apertura; Colaboración; Empatía; Tolerancia; Resolución de conflictos

Fuente: Adaptado de Mateo-Berganza et al. (2019). 


\section{RESULTADOS, DISCUSIÓN Y CONCLUSIONES}

El futuro exigirá estar dotados de habilidades blandas y de capacidad de manejo en entornos digitales. Esto significa que, en cualquier caso, se requiere desarrollar las habilidades blandas para no quedar rezagados en la evolución social y de capacidades técnicas para interactuar en ambientes virtuales (Mateo-Berganza et al., 2019). A todo esto, debe sumarse la capacidad de aprender a aprender y de adaptación, tal como lo propuso Albert Einstein, cuando dijo que "la medida de la inteligencia es la capacidad de cambiar".

Para superar el impacto nocivo y las barreras que impone la COVID-19 en la educación peruana, sobre todo la pública, el Perú ha logrado implementar mejoras en su sistema educativo, que incluyen la implementación de herramientas virtuales de acceso masivo (Zoom, Webex de Cisco, Teams de Microsoft). Del mismo modo, el Ministerio de Educación ha dictado normas para facilitar los traslados de estudiantes a instituciones educativas públicas al no tener capacidad para pagar las pensiones en colegios privados.

Dentro de las herramientas virtuales que destacan, se encuentra la plataforma de aprendizaje virtual "Blackboard Learn", que contiene herramientas interactivas, tales como foros temáticos o de discusión, documentos compartidos, anuncios, actividades individual grupales, etc., además del "Blackboard Collaborate" para clases en vivo. De este modo, se convierte en una herramienta potente porque "el diseño de las actividades de aprendizaje, se basa en las potencialidades de las herramientas info-comunicacionales [...], evaluaciones online, lo que permite un encuentro virtual docente-estudiantes y estudiantes-estudiantes del curso para el trabajo cooperativo, la discusión y el intercambio" (VásquezPajuelo, 2019).

Se concluye en que, se debe perfeccionar las habilidades blandas en el manejo de las propias cualidades socioemocionales, para ser parte de una generación de profesionales capaces de tener un desenvolvimiento emocional armonioso, que incluya momentos de alta presión laboral. La educación superior es partícipe de promover entre la comunidad educativa, en especial entre los estudiantes, la expansión de las mismas, que potencian la interacción de uno con el otro, fortaleciendo el trato, y mejorando las relaciones y el control emocional en el ambiente laboral, profesional y personal.

\section{REFERENCIAS}

Aguerrevere, G., Amaral, N., Bentata, C., \& Rucci, G. (2020, 16 de abril). Frente al Covid-19, desarrollar nuevas habilidades es más importante que nunca [Publicación Web]. Recuperado de https://blogs.iadb. org/trabajo/es/frente-a-covid-19-desarrollar-nuevas -habilidades-es-mas-importante-que-nunca/\#: :text= Pensiones-,Frente\%20a\%20COVID\%2D19\%2C\%20 desarrollar\%20nuevas\%20habilidades,es\%20m\% C3\%A1s\%20importante\%20que\%20nunca\&text= El\%20coronavirus\%20plantea\%2C\%2Odefinitivamente \%2C\%2Oun,el\%2Oempleo\%2Oen\%2Ola\%2Oregi\%C3\%B3n

Ascarza, L. (2020, 25 de marzo). Universidades privadas siguen exigiendo pagos durante cuarentena [Publicación web]. Recuperado de https://wayka.pe/ universidades-privadas-siguen-exigiendo-pensionesdurante-cuarentena/

Así ha afectado el Covid-19 la educación en Colombia. (2020, 30 de abril). Recuperado de https://forbes. co/2020/04/30/actualidad/asi-ha-afectado-el-covid19-la-educacion-en-colombia/

Barrios, E. (2020, 9 de abril). Educación a distancia en tiempo de pandemia. El Peruano. Recuperado de https://elperuano.pe/noticia/94178-educacion-adistancia-en-tiempos-de-pandemia\#: :text=Es\%20 una\%20herramienta\%20que\%20los,televisi\%c3\%B 3n\%2C\%20CD\%2C\%20entre\%20otros.

Duckworth, A., \& Yeager, D. (2015). Cuestiones de medición, evaluación de las cualidades personales distintas de la capacidad cognitiva para fines educativos. https:// www.researchgate.net/publication/276466956_ Measurement_Matters_Assessing_Persona

Flores-Guerra, L. (2018, 10 de diciembre). Cómo desarrollar las habilidades blandas. Recuperado de https://luis floresguerra.com/como-desarrollar-las-habilidades -blandas/ García, P. (2020). Recomendaciones de 
García, P. (2020). Recomendaciones de alimentación y nutrición para la población española ante la crisis sanitaria del COVID-19. Consejo General de colegios oficiales de dietistas-nutricionales, 3.

Goleman, D. (1998). Working withemocional inteligence (1ra. edición ed.). Kairos S. A.

Habilidades blandas: qué son y por qué es importante desarrollarlas (2020, 28 de abril). Recuperado de https://www.universia.net/cl/actualidad/empleo/ habilidades-blandas-que-son-que-importantedesarrollarlas-1078831.html

Hurtado Talavera, F. J. (2020a) Educación, sociedad e ideología: La trilogía imperante del siglo xxi. CIEG, Revista Arbitrada del Centro de Investigación y Estudios Gerenciales, (42), 138-149. Recuperado de http://www.grupocieg.org/archivos_revista/Ed.42(138149)\%2OFrank\%20Junior\%2OHurtado\%20Talavera_ articulo_id602.pdf

Hurtado Talavera, F. J. (2020b). La educación en tiempos de pandemia: Los desafíos de la escuela del siglo XXI. CIEG, Revista Arbitrada del Centro de Investigación y Estudios Gerenciales, (44), 176-187. Recuperado de http://www.grupocieg.org/archivos _revista/Ed.44(176-187)\%2OHurtado\%2OTavalera_ articulo_id650.pdf

Inga Arias, M. G. (2020, 23 de abril). La educación universitaria pública en tiempos de coronavirus [Publicación Web]. Recuperado de https://vrip. unmsm.edu.pe/la-educacion-universitaria-publica -en-tiempos-de-coronavirus/

Izquierdo, R. (2020, 31 de octubre). La OMS desvela las secuelas que tiene la Covid-19. [Publicación Web]. Recuperado de https://as.com/diarioas/2020/10/31/ actualidad/1604152330_708966.html

León-Pérez, F., Bas, M. C., \& Escudero-Nahón, A. (2020). Autopercepción sobre habilidades digitales emergentes en estudiantes de Educación Superior. Comunicar. Media Education Research Journal, 28(1), 91-101. doi:10.3916/C62-2020-08

Mateo-Berganza, M., Buenadicha, C., Bustelo, M., Duryea, S.,
Heredero, E., Rubio-Codina, ... Becerra, L. (2019). Habilidades del siglo 21: Desarrollo de habilidades transversales en América Latina y el Caribe [Publicación del BID]. Recuperado de https:// publications.iadb.org/es/habilidades-del-siglo-21desarrollo-de-habilidades-transversales-en-america -latina-y-el-caribe

Marrero, O. (2018). Habilidades blandas: Necesarias para la formación integral del estudiante universitario. Revista Científica Ecociencia, 18.

Murnane, R., \& Levy, F. (1996). Teaching the new basic skills. Free Press.

Newman, K. S., Chutes \& Ladders, (2006). Navigating the Low-wage Labor Market. Harvard University Press, ISBN 0674023366, p. 351.

Piñero, M. L., Esteban, E. R., Rojas, A. R., \& Callupe, S. F. (2021). Tendencias y desafíos de los programas de posgrado latinoamericanos en contextos de COVID-19. Revista Venezolana de Gerencia (RVG), 26(93), 123-138. doi:10.52080/rvg93.10

Rodriguez Siu, J. L., Rodriguez Salazar, R. E. \& Fuerte Montaño, L. (2021). Habilidades blandas y el desempeño docente en el nivel superior de la educación. Propósitos y Representaciones, 9(1), e1038. DOI: http://dx.doi. org/10.20511/pyr2021.v9n1.1038

Sistema de Información de Tendencias Educativas en América Latina. (2020). Sistematización de respuestas de los sistemas educativos de América Latina a la crisis de la COVID-19. Recuperado de https: //www.siteal.iiep.unesco.org/respuestas_educativas_ covid_19

Siqueira, C. (2017). 5 habilidades blandas fundamentales para triunfar en la actualidad. Universidad de México. https://www.universia.net/mx/actualidad/ empleo/5-habilidades-blandas-fundamentales-triunfar -actualidad-1154123.html

Vásquez-Pajuelo, L. (2019). Aprendizaje online: satisfacción de los universitarios con experiencia laboral. Review of Global Management, 5(2), 28-43. https://doi.org/ 10.19083/rgm.v5i2.1234 